\title{
A Remarkable Pharynx Fibroma
}

\begin{tabular}{|l|l|}
\hline A.P. & Smits \\
\hline In August 1958 a retro-tonsillar compact-elastic tumor was found in a 2 year-old boy, following
\end{tabular}

In August 1958 a retro-tonsillar compact-elastic tumor was found in a 2 year-old boy, following angina, on the left side. The left tonsil was pushed away to the right and forwards. The bloodformula was normal, the sedimentation rate 6/17. The patient had difficulty in swallowing and the food passed through the nose. In September the tumor increased to such a degree that tracheotomy was necessary. Several biopsies were taken and on October 9 the diagnosis nasopharyngeal fibroma rich in cells was established. Because of the rapid growth X-ray irradiation $1500 \mathrm{r}$ was administered.

On October 29 in the Leyden E.N.T.Clinic, under intubation narcosis, the tumor was removed in toto through the mouth, after exposing the left carotid artery, to enable tying up if necessary. The egg-sized tumor proved to be attached to the left wall of the pharynx on the level of the cervicale I; the deeper parts of the tumor resembled a pure fibroma (Prof, van Bijssel).

Thus the tumor partly presented the structure of a naso-pharyngeal fibroma, but the localisation on such a low level of this kind of tumor has up to now never been described. The very rapid growth of the tumor and the age of the boy are remarkable too. In 70 cases, described by van Handousa (1954), the origin of the naso-pharyngeal fibroma always proved to be above the mouth of the Eustachian tube. Figi is the only one who mentioned a lower localisation. Shaheen (1930) too saw a case of naso-pharyngeal fibroma in a child of this age.

Begarding the histological structure

the question remains if the tumor is a simple fibroma or an atypical naso-pharyngeal fibroma sclerosed by irradiation. Possibly an undesired recurrence may corroborate the diagnosis. 